\title{
PERCEPÇÕES DE ENFERMEIRAS SOBRE A ASSISTÊNCIA PRESTADA A MULHERES DIANTE DO ÓBITO FETAL
}

\author{
Nurses' perception on the assistance provided to women facing fetal death \\ Percepciones de enfermeros acerca de la atención a la muj eres delante de la muerte del \\ feto
}

Camila da Silva Santos ${ }^{1}$

Ana Fátima Carvalho Fernandes ${ }^{4}$
Juliana Freitas Marques²

Ana Ciléia Pinto Teixeira Henriques ${ }^{5}$
Francisco Herlânio Costa Carvalho ${ }^{3}$

Karla de Abreu Peixoto Moreira ${ }^{6}$

\section{RESUMO}

Objetivou-se analisar as percepções de enfermeiras quanto à assistência de enfermagem prestada a mulheres diante do diagnóstico de óbito fetal. Pesquisa com abordagem qualitativa, realizada em uma maternidade-escola de Fortaleza-CE, Brasil, entre setembro e outubro/2009. A amostra constou de nove enfermeiras que atuavam no serviço de gestação de alto risco. Os resultados evidenciaram que o foco da assistência prestada era a oferta de apoio psicológico, através da permissão fornecida às mulheres ou familiares para verem o feto morto. As dificuldades mais citadas estiveram associadas à questão estrutural da maternidade, que não possuía uma enfermaria específica para as mães com diagnóstico de óbito fetal. Ademais, citaram a sobrecarga de trabalho, uma vez que exerciam funções que não integravam os procedimentos de práxis. Destaca-se, portanto, a necessidade de uma assistência diferenciada às mulheres ante o óbito fetal, que abranja, além dos recursos humanos, questões estruturais dos serviços de saúde.

Palavras-chave: Saúde da mulher. Morte fetal. Cuidados de enfermagem. Pesquisa qualitativa.

\begin{abstract}
This study aimed to assess the nurses' perception on the nursing care provided to women facing the diagnosis of fetal death. Research with qualitative approach carried out in a maternity school in Fortaleza-CE, Brazil, from September to October 2009. The sample was composed of nine nurses who worked in the service of high-risk pregnancy. The results showed that the focus of assistance offered was the provision of psychological support, through the permission given to women or family members to see the dead fetus. The difficulties most frequently mentioned were associated with the structural issue of motherhood that did not have a specific ward for mothers with a diagnosis of fetal death. They also mentioned the heavy workload, once they had functions that were not part of the procedures of praxis. Thus, we emphasize the need for a different assistance to women facing fetal death covering, in addition to human resources, structural issues of health services.
\end{abstract}

Keywords: Women's health. Fetal death. Nursing care. Qualitative research.

\section{Resumen}

El objetivo del estudio fue analizar las percepciones de enfermeras sobre la atención en enfermería a la mujer con diagnóstico de muerte fetal. Investigación cualitativa, realizada en una maternidad escuela de Fortaleza-CE, Brasil, entre septiembre y octubre de 2009. La muestra consistió de nueve enfermeras del servicio de embarazo de alto riesgo. Los resultados mostraron que el enfoque de la asistencia fue la prestación de apoyo psicológico, a través de la autorización fornecida a las mujeres o familiares para miraren el feto muerto. Las dificultades más frecuentes estuvieron asociadas a la estructura de la maternidad, que no tiene sala específica para las madres con diagnóstico de muerte fetal. Citaron, también, la sobrecarga de trabajo, pues actuaban en procedimientos además de la praxis. Se apunta, así, la necesidad de una atención diferenciada a la mujer con muerte del feto que señale, además de los recursos humanos, las cuestiones estructurales de los servicios de salud.

Palabras clave: Salud de la mujer. Muerte fetal. Atención de enfermería. Investigación cualitativa.

\footnotetext{
'Enfermeira da Escola de Saúde Pública do Ceará/ESP. Fortaleza-CE. Brasil. E-mail: kamila.s.s@hotmail.com; ${ }^{2}$ Mestre em Cuidados Clínicos em Saúde pela Universidade Estadual do Ceará/UECE. Docente da Universidade Federal de Alagoas, Arapiraca - AL. Brasil. E-mail: jufm.marques@gmail.com; ${ }^{3}$ Doutor em Obstetrícia pela Universidade Federal de São Paulo/ UNIFESP. Docente da Pós-graduação em Saúde Coletiva da UFC. Fortaleza-CE. Brasil. E-mail: herlaniocosta@uol.com.br; “Doutora em Enfermagem pelo Programa de Pós-Graduação da UFC. Docente da Pós-graduação em Enfermagem da UFC. Fortaleza-CE. Brasil. E-mail: afcana@ufc.br; ${ }^{5}$ Mestranda em Saúde Pública/FAMED/UFC. Bolsista CNPq. Líder do Grupo de Estudos e Pesquisas em Enfermagem Obstétrica/GEPEO. Fortaleza-CE. Brasil. E-mail: anacileiahenriques@hotmail.com; ${ }^{6}$ Doutoranda em Enfermagem pelo Programa de Pós-Graduação da Universidade Federal do Ceará/UFC. Enfermeira obstetra MEAC/UFC e SESA/CE. Docente da disciplina de Saúde da Mulher e do RN da Faculdade Metropolitana da Grande Fortaleza/FAMETRO.Líder do Grupo de Estudos e Pesquisas em Enfermagem Obstétrica/GEPEO. Fortaleza-CE. Brasil. E-mail: karladeabreupm@hotmail.com
} 


\section{INTRODUÇÃO}

A gravidez traz consigo um conjunto de transformações físicas, psíquicas e sociais que envolvem o casal e o contexto em que estão inseridos. É vista como sinônimo de vida e em nenhum momento remete à perda concreta e finita; quando esta ocorre, toda a simbologia da vida é rompida, resultando em marcas profundas e traumáticas nos pais e nas pessoas que vivenciam este momento.

A ocorrência do óbito fetal não tinha, nos tempos antigos, o mesmo significado que tem na atualidade. Na Idade Média, a morte da criança era pouco valorizada, já que esta era vista como um ser sem personalidade. Ao morrer, muitas vezes, não adquiria nome, e quando recebia um nome, era costumeiramente atribuído diretamente a outra criança. Foi a partir do século XIX que a morte da criança começou a ter relevância. As pessoas então conceberam um lugar diferenciado a elas no céu, e algumas vezes eram consideradas por suas mães como anjos ou santos'.

Independente da simbologia da morte do feto, deve-se compreender que o óbito fetal não se trata apenas da morte em si, mas também da frustração relacionada à experiência vital da mulher durante da gestação ${ }^{2}$.

A aceitação da morte é de grande complexidade, e é difícil concebê-la em qualquer etapa da vida, até mesmo na velhice quando a pessoa já cumpriu parte de seu ciclo vital, bem como no início da vida quando o ser nem sequer chegou a existir fora dos limites do corpo da mãe ${ }^{3}$. Desse modo, compreende-se a magnitude das perdas fetais por suas implicações na vida da mãe e da família que aguardaram ansiosamente o momento único de encontro com o bebê, sonho interrompido pelo diagnóstico do óbito fetal.

Conceitua-se óbito fetal como aquele ocorrido a partir da $22^{\mathrm{a}}$ semana completa de gestação, ou 154 dias, ou de fetos com peso igual ou superior a $500 \mathrm{~g}$ ou estatura a partir de 25 $\mathrm{cm}$. Nestas condições, é obrigatória a emissão de Declaração de Óbito e, consequentemente, de Registro Civil para sepultamento, procedimentos que oficializam a perda fetal e podem facilitar a vivência da perda e do luto por parte da mulher e sua família?2.

Destaca-se que em 2009 ocorreram 23.580 casos de óbito fetal no Brasil. No mesmo ano, ocorreram no Ceará 799 casos; destes, 354 em Fortaleza 4 .

Apesar de aparentemente ser um número pequeno diante da magnitude do número de partos que resultam em fetos viáveis, cogita-se e se busca analisar o componente emocional que acompanha a ocorrência deste evento, além das consequências que ele pode acarretar para a vida da mãe e da família e, principalmente, para aqueles responsáveis pela assistência à mãe diante do óbito fetal mediante a atuação adequada a fim de amenizar o sofrimento daqueles que vivenciam esta situação.
A morte fetal é um evento psicologicamente traumático para a mulher e sua família. As perdas fetais são eventos estressantes que podem ocasionar sérios efeitos a longo prazo. Um estudo que avaliou o impacto de perdas fetais anteriores na gravidez subsequente revelou altos níveis de angústia nas mães, com sentimentos que oscilavam entre o medo e a esperança ${ }^{5}$.

Outro estudo realizado com mulheres com história de desfechos obstétricos adversos, que objetivou avaliar a qualidade de vida e a frequência de ansiedade e depressão nestas, encontrou que aquelas com história de abortamentos recorrentes, óbito fetal, parto pré-termo ou óbito neonatal precoce parecem ter pior qualidade de vida e maior frequência de sintomas de ansiedade e depressão do que mulheres sem história de eventos adversos na gestação ${ }^{6}$.

Com base nos estudos, salienta-se a importância de uma conduta diferenciada na assistência à mulher diante do óbito fetal, visto que estas mulheres tendem a estar mais suscetíveis a distúrbios psicológicos que podem interferir no processo de assimilação do luto e do retorno às suas atividades habituais, já que a morte gera uma enorme sensação de vazio e dor, que será amenizada com o passar do tempo, conforme a perda for sendo elaborada pela família. Porém, as lembranças serão perenes, por todo o mundo de imaginação e de idealização criado durante a gravidez. É importante salientar que 0 processo de assimilação da perda pode variar de pessoa para pessoa ${ }^{3}$.

Após a perda, emergem sentimentos, principalmente por parte da mulher, de culpa, de impotência e de fracasso, por não ter conseguido levar adiante a gravidez. Além disso, é muito frequente a mulher ou o casal buscarem explicações ou fatos que aconteceram durante a gravidez para justificar 0 momento em que se encontram ${ }^{3}$.

À medida que a mulher vai tomando consciência da perda do bebê, muitos são os sentimentos revelados de forma verbal e não verbal: frustração, decepção, revolta, tristeza, culpa e choro ${ }^{7}$.

Assim, cabe aos profissionais de saúde, inseridos nesse contexto, a ofer ta de uma assistência adequada, humanizada e holística a essas mulheres com diagnóstico de óbito fetal, além de assistência médica, suporte emocional para enfrentar esse momento da vida tão difícil. Contudo, nem sempre a equipe que lida com essas situações encontra-se preparada para tal, tendo pouca habilidade para lidar com o sofrimento e os sentimentos presentes'.

Evitar falar do assunto, passar a responsabilidade de informar o óbito fetal às outras categorias profissionais como psicólogo e assistente social, focar apenas 0 aspecto biológico e renegar o psicológico, entre outras atitudes, demonstram 0 despreparo dos diferentes membros da equipe de saúde para uma adequada assistência à paciente em face do óbito fetal ${ }^{2}$.

A maioria dos trabalhos científicos sobre perdas fetais no campo da saúde pública tem contemplado estudos com 
abordagem quantitativa, buscando pesquisar apenas as causas biológicas, os fatores de risco social ou psicossocial associados às perdas fetais ${ }^{7}$. Poucos têm dado ênfase ao dinamismo de sentimentos maternos diante de perdas fetais ou perinatais e à atuação dos profissionais de saúde diante disso, sobretudo os profissionais de enfermagem.

Estudos têm analisado as percepções das mães diante da perda fetal, ${ }^{1,7}$ porém poucos analisam a percepção do enfermeiro, principal prestador de cuidados destas mulheres e que, por isso, deve estar preparado para acolhê-la e ajudá-la na assimilação da perda, contribuindo com seus conhecimentos e atitudes humanas e profissionais, com vistas a minimizar a dor da mãe e da família.

Diante do exposto, surgiram as seguintes questões norteadoras do estudo: como acontece a assistência de enfermagem prestada a mulheres com diagnóstico de óbito fetal? Ocorre um cuidado diferenciado a essas mulheres com diagnóstico de óbito fetal?

Com o propósito de responder a estes questionamentos, este estudo objetivou analisar as percepções de enfermeiras quanto à assistência de enfermagem prestada a mulheres diante do diagnóstico de óbito fetal.

\section{METODOLOGIA}

Realizou-se um estudo do tipo descritivo-analítico com abordagem qualitativa, pertinente neste caso, visto que se aplica ao estudo da história, das relações, das representações, das crenças, das percepções e das opiniões, produtos das interpretações que os humanos procedem a respeito de como vivem, constroem seus artefatos e a si mesmos, sentem e pensam ${ }^{8}$.

Optou-se por este método uma vez que permite desvelar processos sociais pouco conhecidos referentes a grupos particulares ${ }^{8}$. Logo, tratando-se de temática ainda pouco abordada na literatura e que enfatiza processos complexos envolvendo a própria ótica dos enfermeiros sobre seus processos de trabalho na assistência à mulher com diagnóstico de óbito fetal, configura-se a aplicabilidade do método.

0 estudo foi realizado em um Hospital Maternidade de referência do Estado do Ceará, localizado em Fortaleza, em unidade de atendimento às gestações de alto risco, sendo atendidas neste setor também mulheres com diagnóstico de óbito fetal. 0 estudo foi aprovado pelo Comitê de Ética em Pesquisa da instituição sob parecer $n^{0}$ 062/09.

Contribuíram com o estudo nove enfermeiras que prestavam assistência nessa unidade e que aceitaram participar voluntariamente através da assinatura do Termo de Consentimento Livre e Esclarecido, sendo consentida a gravação de entrevistas semiestruturadas com as seguintes questões norteadoras: Como acontece a assistência de enfermagem prestada a mulheres com diagnóstico de óbito fetal? Ocorre um cuidado diferenciado a mulheres com diagnóstico de óbito fetal? Quais as contribuições e as dificuldades encontradas para se desenvolver uma assistência diferenciada a essas mulheres?

Os dados qualitativos foram analisados pela Técnica de Análise de Narrativas, na qual considera a narrativa da entrevistada em detalhes e de acordo com seu contexto de vida ${ }^{9}$.

Para preservar a identidade das participantes, foram utilizados nomes de rainhas, por se tratarem de mulheres que foram grandes exemplos de cultura, liderança, poder e sabedoria para a sociedade: Rainha Elizabeth Bowes-Lyon, Rainha Esther, Rainha Anastácia, Rainha Balkis, Rainha Cleópatra, Rainha Nefertiti, Rainha Catarina Médicis, Rainha Sofia e Rainha Elizabeth II.

\section{RESULTADOS E DISCUSSÃO}

A partir dos discursos das entrevistadas foram evidenciadas 11 unidades de significação: apoio emocional; preparo dos profissionais; importância da equipe multidisciplinar; descrição dos procedimentos realizados; disponibilização de mostrar o feto morto para mãe ou família; facilitação da entrada do acompanhante; ausência de enfermarias específicas para mulheres com óbito fetal; déficit de acomodação para acompanhantes; fluxo de pacientes; superlotação das enfermarias; e sobrecarga de trabalho. Após encontradas as unidades de significação, foram estabelecidas as unidades de sentido: assistência às mulheres com foco no emocional; contribuições para uma assistência diferenciada diante do óbito fetal: avanços e desafios; e aspectos organizacionais e estruturais da maternidade.

Pontuaram-se alguns discursos extraídos das entrevistadas a fim de apresentar os principais aspectos relatados por elas, os quais foram discutidos à luz da literatura pertinente.

\section{Assistência às mulheres com foco no emocional}

0 suporte psicológico foi referido pelas enfermeiras como o principal aspecto a ser enfatizado na assistência a estas mulheres, pois visa proporcionar maior confor to nesse momento, já que o estado emocional destas encontra-se em desequilíbrio.

Entretanto, apesar de os discursos enfatizarem o suporte psicológico, as enfermeiras afirmaram que não se via a realização de um cuidado especializado em outros aspectos, o que foi representado como um sentimento de impotência para as profissionais diante do óbito fetal:

Em primeiro lugar éo apoio psicológico, [...] quando nós temos psicólogos na casa, nós entramos em contato com a psicóloga, ela vem conversa com a paciente. Essa paciente, todas elas apresentam um baixo astral, um sistema emocional abalado, e os cuidados prestados demais éigual às outras. (Rainha Balkis) 
[...] a gente tenta conversar, mostrar novas chances que ela tem pela frente de engravidar novamente, que cada coisa que acontece não acontece por acaso, então a gente vai tentar lidar com a habilidade de comunicação. É o que a gente da enfermagem faz porque a gente não pode fazer mais do que isso. (Rainha Anastácia)

Apesar de perceberem a importância do suporte emocional, observa-se nos discursos o despreparo destes profissionais em lidar com o óbito fetal, renegando por vezes suas atribuições a outros profissionais, os quais consideram mais preparados para lidar com a situação.

Dessas dificuldades surge a esquiva em lidar com pacientes nessas condições. Logo, observa-se o medo de informar a mulher sobre o diagnóstico e a ênfase apenas nos aspectos biológicos da situação, evitando abordar o tema sob a ótica emocional e suas repercussões psicológicas, que podem, se negadas, acarretar danos diversos não apenas para a mãe, mas também para a família que sofre a perda fetal?

Em contrapartida, as enfermeiras assumiram a necessidade de maior preparo da equipe de enfermagem para lidar com o óbito fetal. Nos discursos, relataram que até conseguiam realizar essa assistência, porém com fracassos, pois, para elas, enfermeiros e médicos não estão preparados, em alguns casos, como os psicólogos estão, em relação à conversa, escuta e preparação da família.

[...] não existe um preparo, eles (médicos) não estão preparados, eles não são mesmo, e a gente (enfermeira) não tem o suporte da psicóloga, entendeu. Então muitas vezes a gente faz esse papel, mas a gente não tem esse treinamento pra lidar como a psicóloga tem, da escuta, do preparo da familia. [...] se fosse uma coisa mais organizada, mais estruturada, e uma equipe treinada pra esse tipo de clientela, eu tenho certeza que iria ser diferente. (Rainha Anastácia)

Corrobora-se que a equipe de saúde, em alguns casos, não se encontra preparada para lidar com estas situações, de modo que alguns membros o fazem de maneira mais adequada do que outros, já que isso poderá depender da compaixão e de características pessoais de cada um ${ }^{10}$.

Em relação ao óbito fetal, muitas mulheres apresentam problemas psicossociais durante o período do luto, fato que afeta não só a mãe, mas também o pai da criança. Por isso, é importante que haja o melhor preparo do profissional de saúde para com as mulheres com diagnóstico de óbito fetal, visto que, quanto mais precoce a intervenção realizada, maiores são as chances de minimizar o trauma da perda ${ }^{11}$. Enfatiza-se a importância de o enfermeiro assumir seu papel na assistência a estas mulheres e fundamentar suas ações nas recomendações conhecidamente benéficas, a fim de minimizar o transtorno emocional por qual se encontram a gestante e sua família.

Devido a intensa carga emocional sofrida pelas mulheres com diagnóstico de óbito fetal, ressalta-se o desenvolvimento de ações específicas voltadas para estas mulheres, ações que devem ser realizadas por todos os profissionais que prestam assistência a pacientes nessas condições. Nesse sentido, dentre as ações realizadas, uma enfermeira baseou-se no suporte psicológico, na fala e na escuta.

\section{[...] na verdade a minha assistência com a paciente é mais com o lado psicológico porque pouca coisa a gente da enfermagem tem o que fazer, é mesmo mais conversar, ouvir (Rainha Anastácia).}

Porém, mesmo com a maior facilidade de aproximação destas mulheres, o que se visualiza é que existem dificuldades, tanto da paciente em manifestar seus sentimentos e dúvidas, como da equipe em promover o diálogo?

A condição de fragilidade em que as mulheres se encontram foi apontada como um fator de facilitação à aproximação destas mulheres, que permitem o desenvolvimento de ações terapêuticas.

As dificuldades a gente não encontra não, por que ela está muito carente e a gente não tem dificuldade pra chegar até essa paciente. (Rainha Elizabeth Bowes-Lion)

Em vista disso, torna-se importante aprender a escutar a dor e o sofrimento com o intuito de se evitar possíveis transtornos psicológicos, pois sufocar a dor é ainda pior, porque esta provavelmente se manifestará de outra forma, tendendo a tornar-se crônica e a crescer, caso reprimida ${ }^{12}$.

Nesse contexto, o processo de assimilação da perda inicia-se logo no momento do diagnóstico do óbito fetal; neste momento, é fundamental pensar na maneira de transmitir o diagnóstico, assim como no acompanhamento que será dado a esta mulher e a sua família até a alta hospitalar. Em consequência, a equipe que trabalha adequadamente tem papel crucial na reorganização emocional da mulher que vivenciou a perda fetal ${ }^{13}$.

\section{Contribuições para uma assistência diferenciada diante do óbito fetal: avanços e desafios}

As enfermeiras ressaltaram nos discursos que, para a realização de uma assistência diferenciada, faz-se necessária a implantação da Sistematização da Assistência de Enfermagem (SAE), maneira pela qual é possível individualizar a assistência de enfermagem voltada para a necessidade da cliente.

[...] assistência diferenciada estaria começando com a implementação da sistematização da assistência 
de enfermagem [...], visto que nós temos pessoas com bastante formação, com mestrado, doutorado [...] tem inúmeras enfermeiras com essas titulações Então bastaria que tivesse interesse, alguém que pudesse esta puxando esse cordão, esse grupo, pra estimular pra essa tentativa [...] (Rainha Nefertiti)

No entanto, apesar de ressaltar a importância da sistematização da assistência diante do óbito fetal, o discurso da enfermeira sinalizou a carência de articulação entre a formação profissional prática e a acadêmica, o que é apontado pelo baixo impacto da academia para a transformação dos serviços de saúde na prestação da assistência em geral ${ }^{14}$.

A presença de profissionais com altas titulações acadêmicas e, consequentemente, relacionadas a um maior conhecimento sobre determinadas áreas não implica a aplicação destes conhecimentos nas práticas dos serviços de saúde, o que foi identificado como pouco interesse dos profissionais em realizar uma assistência adequada a determinados contextos.

\section{[...] eu vejo que há um desinteresse dos profissionais em implementar a sistematização da assistência de enfermagem, que seria uma forma de está dando uma assistência diferenciada e individualizada para essa mulher. (Rainha Nefertiti)}

Outra contribuição bastante citada pelas enfermeiras foi a possibilidade de facilitarem determinados procedimentos, como o direito de as mulheres ou familiares de verem o feto morto, possibilitando a comunicação com o serviço social.

\section{Outra contribuição é que muitas vezes elas (mães) pedem pra ver o bebê morto, então a gente liga pro serviço social, porque sempre tem essa ponte como serviço social [...] e quando a mãe quer ver, mostra pra mãe, ou quando a mãe não quer ver, mostra pra familia, por que às vezes eles querem ver... é difícil você lidar com essa perda que você tava criando tudo. (Rainha Anastácia)}

É imprescindível o preparo da enfermagem para conduzir adequadamente o momento do nascimento do feto morto, destacando entre suas ações a necessidade de mostrar o bebê para a mãe. A mãe precisa conhecer seu filho, tocálo, dar um nome, pois isso irá auxiliá-la no processo de elaboração da perda e do enfrentamento da realidade ${ }^{3}$. Segurar o bebê como se estivesse vivo, tirar fotografias e dar um nome são também medidas que facilitam o processo de assimilação da perda ${ }^{12}$.

A imagem incompleta do filho tende a dificultar 0 processo de luto, sendo assim fundamental que os pais vejam 0 filho morto. Orienta-se que se, no primeiro momento, os pais ficarem indecisos ante esta atitude de reconhecimento do bebê, nova oportunidade the seja oferecida nas horas subsequentes, quando o medo e o temor já podem ter sido superados ${ }^{2}$.

Porém, o despreparo por parte dos profissionais, além do medo de intensificar a dor sentida pela perda do bebê, motiva a frequente atitude de afastar os pais da situação de morte, o que contribui para a intensificação dos problemas emocionais enfrentados no período subsequente à alta hospitalar ${ }^{1}$.

A importância da presença de redes de apoio, como a família, os amigos e o companheiro, também foi relatada pelas enfermeiras, que referiram tentar facilitar a entrada dos familiares com a finalidade de fornecer uma assistência diferenciada para a mulher em face do óbito fetal.

A gente tenta facilitar a entrada dos familiares, do companheiro, é [...] mesmo quando ela ainda não tem, no momento, não tem o direito do acompanhante a gente tenta favorecer essa entrada da família, essa comunicação através do serviço social. (Rainha Esther)

Em um estudo recente, as mães citaram como importantes pilares dessas redes de apoio a família e a igreja, e, de maneira pouco expressiva, a figura do psicólogo contrariamente, esta última foi a mais ressaltada pelas enfermeiras participantes deste estudo.

0 apoio da família é evidenciado devido à situação em si, na qual há a necessidade de tomada de decisão no processo burocrático e o enfrentamento social que as mulheres terão de imediato à perda. Por isso, a relevância da valorização da presença da família pelas enfermeiras².

No entanto, apesar das contribuições relatadas, as enfermeiras destacaram nitidamente a carência de um direcionamento de procedimentos específicos para as mulheres com diagnóstico de óbito fetal.

Olha, não tem um cuidado assim específico a essas mulheres, na nossa unidade de gestante de alto risco e puérperas de alto risco, todas elas são cuidadas de uma mesma forma. Não tem uma questão assim, não tem uma assistência específica pra ela nessa ocasião, são esses cuidados gerais, que nos fazemos pra outras pacientes também. (Rainha Esther)

As enfermeiras relataram que, por vezes, não conseguiam oferecer uma assistência diferenciada para estas mulheres, visto que havia a sobrecarga da jornada de trabalho, em que tinham que realizar várias outras atividades e até mesmo procedimentos de outros profissionais, como coleta de amostras para exames laboratoriais, pois muitas vezes havia apenas uma enfermeira para vários pacientes. 
[...] tem aqui a enfermeira que passa o plantão, mas tem a enfermeira que toma conta de várias coisas, quarenta e tantos pacientes; então, o tempo da gente conversar, explicar, é o que éfundamental, dar apoio ao paciente, que éo que eu vejo que elas mais necessitam [...]. [...] a nossa demanda é muito grande e às vezes não dá pra acompanhar de perto. (Rainha Sofia)

A sobrecarga de trabalho das enfermeiras muitas vezes motiva que diversas orientações importantes não sejam fornecidas, o que pode suscitar nas mulheres um sentimento de abandono e desrespeito. É importante que todas as informações a respeito do parto sejam fornecidas claramente e que a mulher seja preparada pela equipe para vivenciar realisticamente esse momento?.

Ao informar o diagnóstico de morte fetal, espera-se que 0 auxílio à cliente se caracterize pela neutralidade no esclarecimento sobre os procedimentos diagnósticos e terapêuticos ${ }^{15}$, o que muitas vezes é dificultado pelo excesso de atribuições dadas às enfermeiras, consoante justificativas das participantes.

\section{Aspectos organizacionais e estruturais da maternidade}

Outro aspecto relatado pelas enfermeiras residiu nas deficiências estruturais da maternidade, que não possuía uma enfermaria específica para as mães com diagnóstico de óbito fetal e para o pós-parto destas, o que também dificultava a assistência. Mulheres com este diagnóstico presenciavam outras mulheres com filhos, o que poderia ocasionar desequilíbrio emocional maior.

Eu acho que, de imediato, a gente poderia ver a acomodação; aqui as pacientes com óbitos fetais, elas ficam nas enfermarias onde tem vaga e, assim, ficam junto com parturientes. Aquilo dali se torna mais incômodo pra elas, a gente nota elas afastadas por conta disso, saber que elas vão ter um feto mor to e ali ela gestante, um feto morto na barriga e vendo aquelas outras vizinhas tudo com seus bebês. (Rainha Catarina Médicis)

A necessidade de mudança na organização da unidade apontada pela entrevistada reflete que a assistência de enfermagem não termina com o óbito fetal. É de responsabilidade do enfermeiro prestar cuidados diferenciados ao que vivenciam o luto, sendo necessário rever conceitos sobre a existência, a vida e a morte, a fim de que se possa, em cada situação, agir de forma adequada e humanizada ${ }^{16}$.

Recomenda-se que a decisão acerca do local de permanência após o parto deva ser discutida com a própria mãe. Para algumas, permanecer sozinha seria muito importante, há as que prefeririam a companhia de outras mães e seus bebês². É significante, portanto, dialogar com a mulher sobre estas possibilidades, porém as dificuldades se encontraram na indisponibilidade de escolha pelas mulheres.

Ressalta-se, por meio do discurso de uma enfermeira, a necessidade de adequar à estrutura hospitalar para também proporcionar maior conforto ao acompanhante, pois neste momento é importante que a paciente tenha o apoio também da família.

Eo conforto do acompanhante, nós não temos, nós estamos no hospital com enfermaria de seis leitos, e não tem aquele conforto pra aquele acompanhante ficar, é possivel, mas sem muito conforto [...] (Rainha Esther)

0 pai do bebê tem sido descrito como uma figura fundamental ${ }^{17}$. Algumas vezes, cabe ao profissional simplesmente servir de apoio, para que a própria família se ampare mutuamente. É importante conversar com as pacientes, investigar como está ocorrendo o seu contato com a família, procurando reforçar o apoio familiar. Também é importante conversar com o pai do bebê falecido, para transmitir apoio e reforçar a união do casal nesse momento. 0 pai, em geral, também está sofrendo bastante e, frequentemente, ainda, temendo pela saúde de sua esposa/ companheira. É necessária a união do casal, para que se apoiem mutuamente e encontrem forças para enfrentar a perda. Nos momentos em que falta essa proximidade, então, o sofrimento costuma ser potencializado e podem surgir figuras substitutivas'.

As deficiências estruturais da maternidade citadas pelas enfermeiras foram consideradas empecilhos para a realização de uma assistência de qualidade às mulheres e aos seus familiares que vivenciaram a difícil situação do óbito fetal, abrangendo as dificuldades além das próprias ações da enfermagem.

As equipes de saúde que lidam com as intercorrências presentes no ciclo gravídico-puerperal precisam estar adequadamente preparadas para tolerar 0 sofrimento psíquico, presente nas mulheres que sofrem perda gestacional. Para que isso aconteça, as instituições devem proporcionar a capacitação adequada aos profissionais, através de formação constante. Em geral, na memória de mulheres que são atendidas satisfatoriamente no contexto da perda gestacional, permanecem boas recordações do atendimento humanizado oferecido pela equipe ${ }^{18}$.

Faz-se necessária uma mudança de paradigma nessa missão de atender pessoas, reforçando a necessidade de humanização do atendimento nos serviços de saúde ${ }^{8}$, em especial àquelas mulheres que se encontram diante de condições graves e que carregam fortemente as consequências psicológicas de sua condição. 


\section{CONSIDERAÇÕES FINAIS}

Com base na análise das entrevistas, pôde se constatar que o foco da assistência prestada por enfermeiras às mulheres com diagnóstico de óbito fetal consiste em fornecer suporte psicológico no momento em que a mulher se encontra em desequilíbrio emocionalmente, cuja principal função relatada pelas enfermeiras participantes seria na escuta e no falar, para amenizar o momento de profunda tristeza dessas mulheres.

0 óbito fetal representa um episódio dos mais frustrantes e de difícil elaboração na vida de uma mulher, e o enfermeiro, ao se deparar com esse acontecimento, deve estar preparado para assistência devida. Percebeu-se nos discursos sentimentos de impotência dos enfermeiros diante do óbito fetal e uma consciência de incompletude acerca da assistência prestada em relação ao que deveria ser realizado.

Para o enfermeiro prestar um cuidado com qualidade, torna-se necessário o adequado conhecimento teórico-prático sobre a situação de perda fetal, bem como sensibilidade para compreender as principais necessidades da cliente e realizar as intervenções conforme o estabelecido por seus anseios. Vale ressaltar que seria interessante a construção de um plano de cuidado específico para as mulheres com óbito fetal, visto que elas necessitam de uma assistência diferenciada e individualizada; neste sentido, ressaltou-se o papel da SAE como instrumento norteador das ações a serem realizadas, fundamentando uma atuação de qualidade dos enfermeiros e sua equipe.

Porém verificou-se que as entrevistadas relegam a implantação da SAE às profissionais que têm maiores titulações, não reconhecendo este instrumento como uma ação fundamental do enfermeiro qual seja o grau de formação. É preciso que estes profissionais se instrumentalizem com a SAE não apenas reconhecendo seu valor no embasamento a uma assistência de qualidade, mas assumindo que não se admite a atuação do enfermeiro sem esse fundamental direcionamento.

Assim, o enfermeiro deve ter como contribuição participar e realizar capacitações da equipe, através do fornecimento das informações necessárias, para que 0 atendimento a mulher ocorra de forma holística, não se limitando apenas aos cuidados técnicos de enfermagem, mas abordando todo o contexto biopsicossocial da mulher e da família que está vivenciando o óbito fetal.

Nesse contexto, um atendimento diferenciado requer das instituições de saúde também estruturação física adequada relacionada à possibilidade de escolha das mulheres em permanecerem ou não em enfermarias conjuntas e à existência de espaços adequados para expressão dos sentimentos, além de adequada acomodação do acompanhante para que ambos se auxiliem no processo de elaboração da perda. Atitudes como essas são importantes por colocarem a paciente e sua família no caminho da elaboração adequada do luto pela perda da criança.
Todavia, destacou-se a realidade da sobrecarga de trabalho dos enfermeiros como fator impeditivo à realização de uma assistência satisfatória, o que reforça a necessidade de otimizar a distribuição de mulheres por profissional a assistilas, além de melhor distribuição das atribuições dos enfermeiros, para que possam assistir de forma integral, com o intuito de minimizar a dor daquelas que vivenciam o óbito fetal.

Reconhecem-se as limitações do estudo, visto que este foi realizado com profissionais que atuam rotineiramente na assistência a pacientes de alto risco gestacional, perfil assistido na maternidade, e que, devido à rotina profissional, estes não identificaram uma assistência diferenciada às mulheres diante do óbito fetal quando comparada àquela prestada diante de outras complicações obstétricas, o que pode ter resultado na percepção de impotência diante da questão do óbito fetal como apresentado na fala das entrevistadas.

Porém, mesmo reconhecendo as deficiências percebidas pelas próprias enfermeiras, não se deve relegar a importância da atuação destas profissionais na amenização da dor e do impacto causado na vida das mulheres e seus familiares diante do diagnóstico do óbito fetal, traduzindo um dos grandes preceitos da Enfermagem, que é o cuidar sob toda e qualquer circunstância.

\section{REFERÊNCIAS}

1. Carvalho FT, Meyer L. Perda gestacional tardia: aspectos a serem enfrentados por mulheres e conduta profissional frente a essas situações. Bol Psicol. 2007 jun; 57(126): 33-48

2. Luz AMH, Santos ES, Mendes SMA, Agostini SM. Feto morto: atuação da enfermeira frente ao sentimento materno. Rev Bras Enferm. 1989 jan/ dez; 42(1/4): 92-100.

3. Ampese D, Perosa G, Haas RE. A influência da atuação da enfermagem aos pais que vivenciam a morte do feto. Bioethikos. 2007 jan; 1(2):7077.

4. Instituto Brasileiro de Geografia e Estatística- IBGE. Diretoria de pesquisas. coordenação de população e indicadores sociais. Estatísticas do registro civil. Rio de Janeiro; 2009.

5. Armstrong DS. Impact of prior perinatal loss on subsequent pregnancies. J Obstet Gynecol Neonatal Nurs. 2004 nov/dez; 33 (6): 765-73.

6. Couto ER, Couto E, Vian B, Gregório Z, Nomura ML, Zaccaria R, Passini Junior R. Quality of life, depression and anxiety among pregnant women with previous adverse pregnancy outcomes. Sao Paulo Med J. 2009 jul; 127 (4): 185-89.

7. Santos ALD, Rosenburg CP, Buralli KO. Histórias de perdas fetais contadas por mulheres: estudo de análise qualitativa. Rev Saude Publica. 2004 abr; 38(2): 268-76. 
8. Minayo MCS. 0 desafio do conhecimento: pesquisa qualitativa em saúde. $10^{a}$ ed. São Paulo: Hucitec; 2007.

9. Bauer MW, Gaskell G. Pesquisa qualitativa com texto, imagem e som: um manual prático. $2^{\mathrm{a}}$ ed. Petrópolis (RJ) : Vozes; 2002.

10. Gonçalves MO. Morte e castração: um estudo psicanalítico sobre doença terminal infantil. Psicol Cienc Prof. 2001 mar; 21(1): 31-40.

11. Sutan R, Amin RM, Ariffin KB, Teng TZ, Kamal MF, Rusli RZ. Psychosocial impact of mothers with perinatal loss and its contributing factors: an insight. J Zhejiang Univ Sci B. 2010, 11(3): 209-17.

12. Braga NA, Morsch DS. Quando o bebê morre. In: Moreira MEL, Braga NA, Morsch DS, organizadores. Quando a vida começa diferente: o bebê e sua família na UTI Neonatal. Rio de Janeiro: Fiocruz; 2003. p.157- 69.

13. Safund K, Sjogren B, Wredling R. The role of caregivers after a stillbirth: views

and experiences of parents. Birth Defcts. 2004 jun; 31:132-37.

14. Gonçalves LRR, Nery IS, Nogueira LT, Bonfim EG. 0 desafio de implantar a sistematização da assistência de enfermagem sob a ótica de discentes. Esc Anna Nery. 2007 set; 11 (3): 459-65.

15. Benute GRG, Nomura RMY, Lucia MCS, Zugaib M. Interrupção da gestação após o diagnóstico de malformação fetal letal: aspectos emocionais. Rev Bras Ginecol Obstet. 2006 jan; 28(1): 10-17.

16. Aguiar IR, et al. 0 envolvimento de enfermeiro no processo de morrer de bebês internados em unidade neonatal. Acta Paul Enferm. 2006; 19(2): 131-37.

17. Rodrigues MML, Hogo LAK. Homens e abortamento espontâneo: narrativas das experiências compartilhadas. Rev Esc Enferm USP. 2005 set; 39(3): 258-67.

18. Bortoletti FF. Psicodinâmica do ciclo gravídico puerperal. In: Bortoletti FF, organizador. Psicologia na prática obstétrica: abordagem interdisciplinar. Barueri: Manole; 2007. 